\title{
Un examen pour les instituteurs : le brevet de capacité de l'instruction primaire dans le département de la Somme (1833-1880)
}

An Exam for Primary School Teachers : the primary school teacher's diploma in the department of the Somme, 1833-1880

Eine Eingangsprüfung für zukünftige Volksschullehrer: der Befähigungsnachweis für Elementarschullehrer im Departement Somme 1833-1880

Daniel Toussaint

\section{OpenEdition}

1 Journals

\section{Édition électronique}

URL : https://journals.openedition.org/histoire-education/814

DOI : $10.4000 /$ histoire-education.814

ISSN : 2102-5452

Éditeur

ENS Éditions

\section{Édition imprimée}

Date de publication : 1 mai 2002

Pagination : 75-101

ISBN : 2-7342-0923

ISSN : 0221-6280

Référence électronique

Daniel Toussaint, « Un examen pour les instituteurs : le brevet de capacité de l'instruction primaire dans le département de la Somme (1833-1880) », Histoire de l'éducation [En ligne], 94 | 2002, mis en ligne le 07 janvier 2009, consulté le 20 mai 2021. URL : http://journals.openedition.org/histoireeducation/814 ; DOI : https://doi.org/10.4000/histoire-education.814

Ce document a été généré automatiquement le 20 mai 2021.

(c) Tous droits réservés 


\section{Un examen pour les instituteurs : le brevet de capacité de l'instruction primaire dans le département de la Somme (1833-1880)}

An Exam for Primary School Teachers : the primary school teacher's diploma in

the department of the Somme, 1833-1880

Eine Eingangsprüfung für zukünftige Volksschullehrer: der

Befähigungsnachweis für Elementarschullehrer im Departement Somme

$1833-1880$

Daniel Toussaint

1 L'ordonnance royale sur l'instruction primaire du 29 février 1816 institue, dans ses articles 10 et 11, des brevets de capacité obligatoires pour enseigner et des examens pour leur obtention. Cette création marque l'aboutissement d'une réflexion sur l'enseignement primaire et plus particulièrement sur la mise en place d'un corps d'instituteurs de qualité et compétent, engagée depuis 1810-1811. Le décret du 17 mars 1808 laissait les instituteurs en dehors de l'Université. Seules les fonctions exercées dans le secondaire nécessitaient la possession d'un grade (baccalauréat, licence et doctorat) délivré par les facultés après examen ${ }^{1}$. Cette garantie, l'Université ne l'exigeait pas des maîtres du primaire. Elle se contentait d'un simple contrôle hiérarchique et administratif exercé par les recteurs et les inspecteurs d'académie et du souhait qu'il fût créé des classes normales dans les lycées et collèges "destinées à former des maîtres pour les écoles primaires $»^{2}$ (art. 108 du décret du 17 mars 1808). Le choix qui est fait en 1816 s'inspire donc du modèle secondaire: un examen pour l'obtention d'un diplôme, garant d'un certain niveau de connaissances. Passé devant l'inspecteur d'académie ou un délégué du recteur, cet examen renforce la mainmise de l'État sur les enseignants du primaire et engage un processus d'uniformisation. La frontière avec le secondaire est néanmoins réaffirmée, car le brevet de capacité n'est 
aucunement un nouveau grade universitaire et ne fait donc pas du maître d'école un membre de l'Université.

2 Avec les brevets de capacité, il existe donc désormais des diplômes nationaux, décernés après examen, pour les enseignants du primaire. L'enjeu d'un tel examen est considérable, comme le soulignera François Guizot dans l'exposé des motifs de la loi du 28 juin 1833 : "Il est évident que l'instruction primaire tout entière repose sur cet examen [...]. Supposez qu'on y mette un peu de négligence, ou de complaisance, ou d'ignorance, et c'en est fait de l'instruction primaire $»^{3}$. Vingt ans plus tard, dans une circulaire du 31 octobre 1854, Hippolyte Fortoul rappellera, lui aussi, que «de l'élévation ou de l'abaissement du niveau de cet examen dépend le sort de l'enseignement $»^{4}$.

3 Le vote de la loi du 28 juin 1833 et le règlement du 19 juillet 1833 sur les brevets représentent une étape fondamentale dans l'histoire de ces diplômes. En effet, l'établissement de deux sessions annuelles publiques d'examen organisées autour d'une commission nommée par le ministre, l'obligation faite aux normaliens de se présenter aux épreuves, le renforcement des exigences disciplinaires et la publicité qui accompagne les convocations, font des brevets de capacité une véritable institution. Une culture d'examen s'installe dans le primaire: les maitresses de pension et les maitresses d'étude, les sous-inspecteurs puis les inspecteurs primaires, les directeurs d'écoles normales, les surveillants et surveillantes des salles d'asile ${ }^{5}$, doivent aussi se rendre devant des commissions pour subir un ensemble d'épreuves qui attestent ou non de leurs compétences.

4 Ces examens, dont les archives ont gardé les traces, dessinent un espace social nouveau, créateur de nouvelles hiérarchies, de nouvelles distinctions, de nouveaux enjeux. C'est là un lieu de rencontre entre des acteurs et entre des savoirs, où se fondent les premiers éléments d'une culture primaire ${ }^{6}$. Identifier les acteurs, membres des commissions et les candidats, s'interroger sur le déroulement des sessions, cerner les savoirs tels qu'ils se construisent dans cet espace, analyser les performances des individus, c'est commencer à comprendre la fonction des brevets de capacité, leur rôle dans la définition d'un savoir normé, exigé dorénavant des futurs maitres d'école, et, finalement, leur contribution à l'invention de l'instituteur contemporain. C'est cette fonction que l'étude précise du corpus archivistique du département de la Somme concernant les brevets de capacité peut nous permettre de préciser.

\section{Les acteurs de l'examen}

5 Deux fois par $a^{7}$, au printemps et en été, se tiennent à Amiens, chef-lieu du département de la Somme et chef-lieu d'académie ${ }^{8}$, les sessions d'examen pour l'obtention des brevets de capacité de l'instruction primaire. Candidats et candidates ${ }^{9}$, préalablement inscrits, se présentent alors devant une même commission départementale, composée de sept à quinze membres selon les sessions et présidée le plus souvent par l'inspecteur d'académie, pour y être interrogés sur leur connaissance des disciplines enseignées à l'école et des méthodes d'enseignement. 


\section{Les commissions d'instruction primaire}

6 La première commission d'instruction primaire chargée d'interroger les candidats au brevet dans la Somme a été nommée par un arrêté du recteur de l'académie d'Amiens en date du $1^{\text {er }}$ octobre $1832^{10}$. Elle est composée de cinq membres. La pratique des examens se conformait jusqu'alors à l'ordonnance royale du 29 février 1816, qui confiait à l'inspecteur d'académie ou à un fonctionnaire de l'instruction publique délégué par le recteur le soin d'examiner les candidats. Dans le département de la Somme, celui-ci désignait un représentant dans chacun des cinq arrondissements : l'inspecteur d'académie pour l'arrondissement d'Amiens, les principaux des collèges pour les arrondissements qui en étaient pourvus (Abbeville, Montdidier et Péronne à partir de 1820), plusieurs maîtres de pension assistés parfois du curé cantonal pour les autres (Péronne de 1816 à 1820, Doullens).

7 Pourtant, le dispositif annoncé en 1832 par le recteur n'était pas nouveau, car des commissions d'instruction primaire existaient déjà pour l'examen des candidates au brevet de capacité : d'après la circulaire du 3 juin 1819, le préfet devait former « une commission composée de cinq membres dans le chef-lieu du département [...], chargée d'examiner, sous le rapport de l'instruction, les personnes qui désireront se vouer aux fonctions d'institutrices $»^{11}$ et de délivrer le diplôme ${ }^{12}$. Deux modèles fonctionnaient donc depuis 1819 : l'un, adopté pour les garçons, faisait appel à un examinateur unique appartenant au corps universitaire et l'autre, celui des filles, à une commission de type capacitaire, échappant jusqu'en 1828 au contrôle du recteur ${ }^{13}$. C'est donc ce second modèle qui a été repris localement pour les garçons en octobre 1832. Ainsi, lorsque Guizot, par l'article 25 de la loi du 28 juin 1833, institue officiellement dans chaque département "une ou plusieurs commissions d'instruction primaire, chargée d'examiner tous les aspirants aux brevets de capacité [...] et de faire l'examen d'entrée et de sortie des élèves de l'école normale primaire $»^{14}$, il ne fait que généraliser et officialiser une pratique déjà en vigueur pour les candidates et étendue aux garçons dans certaines académies.

Guizot souligne dans l'exposé des motifs toute l'importance qu'il accorde aux choix des hommes : " Les membres de cette commission devront être, au moins en grande partie, des hommes spéciaux, des gens d'école [...]. Il importe de composer ces commissions d'examen avec la sévérité la plus scrupuleuse et de n'y appeler que des gens versés dans la matière $»^{15}$. C'est le ministre lui-même qui choisit les examinateurs. Finalement, l'organisation des examens pour les garçons et les filles est définitivement unifiée, dans la Somme, par l'application de la circulaire ministérielle relative à l'exécution de l'ordonnance du 23 juin 1836 sur les écoles de filles : celle-ci laisse au recteur le soin de choisir, pour l'examen des aspirantes, entre l'établissement de commissions spéciales et l'utilisation

des «commissions déjà établies pour les instituteurs, dans les chefs-lieux des départements, et auxquelles seraient adjointes des dames inspectrices $»^{16}$.

9 Jusqu'en 1850, les commissions d'instruction primaire sont nommées pour trois ans par le ministre, et ensuite chaque année par le conseil académique, puis par le conseil départemental ${ }^{17}$. Leur composition peut être reconstituée pour le département de la Somme depuis 1833. L'Université y domine largement. Représentant les deux tiers des jurés pour l'ensemble de la période 1834-1880, elle a ainsi la haute main sur les examens. L'inspecteur d'académie, parfois le recteur, assiste aux sessions et les préside 
jusqu'en $1864^{18}$. La présence de l'inspecteur primaire ${ }^{19}$ et celle des sous-inspecteurs primaire ${ }^{20}$ sont systématiques. Surtout, la place du secondaire est essentielle. Le collège royal, devenu lycée en 1848 , constitue ainsi une pépinière d'examinateurs : proviseurs, censeurs, professeurs, aumôniers participent activement aux commissions, représentant environ $27 \%$ des membres repérés dans la Somme pendant la période 1834-1880.

10 Le choix de ces enseignants ne relève évidemment pas du hasard. Lors des premières nominations, en 1833-1834, l'appartenance à la Commission des lettres ${ }^{21}$, dont sont membres les trois professeurs désignés, semble avoir joué un rôle déterminant. Les carrières locales des membres de la commission appartenant à l'Université sont souvent brillantes : entrée au Conseil académique, distinction honorifique, charge des cours communaux publics organisés par la municipalité d'Amiens, nomination comme inspecteur d'académie ou recteur. Les parcours de Lebailly, agrégé de lettres, professeur de rhétorique au collège royal d'Amiens, et d'Allou, chef d'institution, illustrent assez bien ce type de carrière. Ils participent aux travaux des commissions d'instruction primaire, puis entrent dans l'administration universitaire, l'un comme inspecteur d'académie, l'autre comme recteur départemental. Un troisième parcours, celui de Braive, confirme la qualité de l'élément universitaire dans les commissions. Élève de l'école normale de Paris entre 1816 et 1819, agrégé de physique et professeur au collège royal d'Amiens, il en devient proviseur de 1834 à 1845 . C'est à ce titre qu'il participe aux travaux de la commission d'instruction primaire de la Somme de 1836 à 1845. Il est nommé inspecteur d'académie à Bourges en 1845 et finit sa carrière comme recteur $^{22}$.

11 La place du secondaire ne se résume pas au personnel du collège. Il faut ajouter la participation de chefs d'institution et de maîtres de pension entre 1833 et 1850, puis de membres de l'enseignement secondaire libre. C'est donc au total plus d'un examinateur sur trois qui appartient à ce niveau d'enseignement. La place du primaire, en revanche, se réduit pour l'essentiel au corps d'inspection (inspecteurs et sous-inspecteurs). Le personnel de l'école normale est exclu des commissions, sauf nécessité absolue ${ }^{23}$. Dans chaque commission, enfin, sont nommés un ou deux aumôniers, chargés de l'instruction morale et religieuse dans leur établissement.

12 Les membres étrangers au monde enseignant représentent environ le tiers des commissions. Ils appartiennent à la magistrature, aux Églises catholique et réformée, au corps médical et aux grands corps d'ingénieurs. Comment sont-ils choisis? Le ministre fait appel principalement à des personnalités, toutes amiénoises, appartenant à l'élite intellectuelle de la ville, reconnues pour leurs savoirs et leur engagement dans la défense et le développement de l'instruction primaire. Plusieurs examinateurs sont membres des sociétés savantes locales et de la Société d'encouragement de l'instruction élémentaire $e^{24}$, et beaucoup se sont fait connaitre par des publications. On retrouve d'ailleurs les mêmes personnalités dans les différentes commissions d'examen instituées pendant la période. L'appel aux élites résidant au chef-lieu de l'académie ou du département est la règle : les mêmes choix sont effectués dans l'Oise et dans l'Aisne, qui composent avec la Somme l'académie d'Amiens ${ }^{25}$. Une étude des jurys nommés entre 1833 et 1850 dans d'autres centres d'examen confirme la volonté ministérielle de faire appel aux capacités locales ${ }^{26}$. Le rôle des sociétés savantes répond à une logique qui était déjà celle des Lumières. Il s'agit pour le ministre de garantir la qualité des examens, leur caractère irréprochable, en les confiant à ce que l'échelon académique 
ou départemental compte de plus savant, de plus instruit et de plus compétent. Le principe capacitaire, déjà partiellement appliqué à la sphère politique et judiciaire ${ }^{27}$, trouve ainsi un prolongement au niveau de l'instruction primaire.

La loi du 15 mars 1850 n'est pas sans conséquences sur la composition des commissions. Outre les modifications institutionnelles déjà signalées, les membres sont renouvelés dans leur presque totalité en 1851. La création d'une commission d'examen pour l'obtention d'un brevet de capacité de l'enseignement secondaire n'y est peut-être pas étrangère ${ }^{28}$. Quatre des six membres désignés le 27 septembre 1850 pour faire partie de cette nouvelle commission participent aux jurys d'instruction primaire depuis plusieurs années. Ces changements ne peuvent donc s'interpréter comme une rupture politique et idéologique, au contraire, mais ils permettent de nouveaux équilibres. Le poids du secondaire dans les commissions d'instruction primaire se renforce (le tiers des effectifs en 1834-1850; $40 \%$ pour la période 1851-1880), avec une recomposition interne au profit des enseignants du lycée (de $13 \%$ à $22 \%$ ) et des chefs d'écoles libres secondaires, eux-mêmes souvent anciens professeurs du collège royal (de $6,7 \%$ à $11,3 \%)$. La place du primaire, qui se réduit à la présence des inspecteurs primaires d'arrondissement, diminue encore (de 25, $9 \%$ à $17 \%)^{29}$. Quant au rôle des ecclésiastiques, il se renforce avec la nomination d'un ou deux prêtres d'une paroisse d'Amiens et d'un pasteur, adjoint systématiquement aux travaux de la commission, ainsi qu'avec la présence d'un second aumônier, celui de la congrégation de la Sainte Famille $^{30}$. Le renforcement des ecclésiastiques participe à la volonté de retour à l'ordre moral. En revanche, l'appel à des personnalités savantes diminue sensiblement (de $27,6 \%$ à 15,8\%). Les commissions se resserrent ainsi autour du corps des professeurs et des inspecteurs. Le profil des commissions d'instruction primaire devient moins capacitaire et plus professionnel.

Ces changements ne doivent pas masquer, cependant, leur relative stabilité. Le renouvellement des membres se fait lentement. Ainsi, Decaïeu, Rigollot et Pollet sont présents pratiquement sans interruption dans les commissions de 1834 à 1850, l'abbé Crampon de 1858 à 1878, l'inspecteur Bonvallet de 1851 à 1869. De 1864 à 1873, la composition des commissions est pratiquement inchangée. Signalons un cas extrême, celui de Garnier, bibliothécaire, qui siège pendant trente-sept ans, de 1843 à 1880 .

Quant aux femmes, leur présence est limitée. Les jurys sont une affaire d'hommes. Lorsque des dames inspectrices leur sont adjoin-tes, comme le prévoit la circulaire du 13 août 1836, c'est seulement pour les épreuves de travaux d'aiguilles chez les candidates. Cette situation n'est pas modifiée après 1850. Dans la pratique, avant la loi Falloux, leur participation est très irrégulière. En septembre 1848, l'inspecteur primaire de la Somme écrit au recteur qu'« il serait bien à désirer que l'on pût parvenir à décider quelques dames de la ville à venir partager les travaux de la commission: certaines parties du programme sont exclusivement de leur ressort $"^{31}$. En revanche, à partir de l'application de la loi du 15 mars 1850, la présence d'une, deux ou trois inspectrices est constatée dans toutes les sessions. Dans certaines académies deux commissions se maintiennent, une pour les garçons et une pour les filles. C'est à cette dernière que sont évidemment adjointes les examinatrices, les titulaires restant des hommes. 


\section{Candidats et candidates} à plusieurs reprises : environ 7000 pour les premiers et 3000 pour les secondes, de mars 1836 à juillet 1880. Sur ce nombre, ce sont, pour la même période, 3000 brevets qui ont été attribués aux candidats et environ 1650 aux filles. Les taux de réussite, en moyenne de 40 à $45 \%$, masquent des disparités importantes, entre les deux sessions de printemps et d'été, les sexes, les types de brevets obtenus. En juillet ou août les résultats sont supérieurs à ceux de mars. La présence des normaliens explique cette différence. Leurs taux de réussite sont en effet de 75 à $100 \%$. Les candidates, moins nombreuses, réussissent mieux que les garçons, avec des taux souvent compris entre 50 et $60 \%$ et pouvant dépasser $70 \%$ pendant certaines sessions. Les jurys sont-ils moins sévères avec les filles, leur recrutement devenant une priorité, ou sont-elles plus capables que les garçons ${ }^{32}$ ?

Les besoins dans le département de la Somme sont importants. Fortement scolarisé ${ }^{33}$, il bénéficie très tôt de maîtres de qualité. Lorsque Guizot arrive au ministère de l'Instruction publique, tous les instituteurs, sauf exceptions, possèdent un brevet de capacité $^{34}$. En 1850, la totalité des maîtres, au vu des statistiques disponibles, disposent d'un diplôme. En revanche, la situation est différente dans un primaire féminin peu développé. À peine $45 \%$ des institutrices sont brevetées ${ }^{35}$. La situation du personnel enseignant masculin à la veille des lois Ferry a peu changé par rapport au milieu du siècle, hormis l'arrivée d'instituteurs-adjoints dans les écoles ${ }^{36}$. En revanche, le développement de l'enseignement féminin, institutrices titulaires et adjointes, a davantage profité aux congréganistes, non brevetées ${ }^{37}$. Au total, c'est près de $70 \%$ du corps primaire féminin qui échappe, dans la Somme, aux exigences du brevet.

La grande majorité des candidats concourt pour le brevet élémentaire, institué en 1833 et devenu le brevet obligatoire (puis le brevet simple) en 1850. Ce diplôme permet d'enseigner dans les écoles élémentaires. Le brevet supérieur est réservé aux futurs maîtres des écoles primaires supérieures créées par Guizot en 1833. Il est supprimé en 1850. Mais les candidats ont alors la possibilité de compléter leur brevet simple par une partie facultative qu'ils peuvent obtenir après un examen portant sur des disciplines supplémentaires. Ce sont surtout les normaliens qui s'attaquent au brevet supérieur, puis à cette partie facultative, avec des chances de réussite non négligeables ${ }^{38}$.

作 1860 et surtout les années 1870 sont marquées par des changements significatifs. Une hausse du nombre des candidats, associée à une amélioration sensible des résultats pour les deux sessions et à une progression du brevet complet, atteste alors de l'importance des réformes engagées par Duruy ${ }^{39}$, de l'élévation générale du niveau des candidats et d'une demande sociale de plus en plus forte pour l'exercice d'une profession revalorisée. Mais c'est surtout le phénomène de rattrapage constaté au niveau des filles qui nous paraît le plus significatif. L'enjeu est de taille : l'essor traduit la volonté de développer l'enseignement féminin laïque au moment où les congrégations religieuses contrôlent la plupart des écoles de filles du département de la Somme et alors que les maîtresses congréganistes sont dispensées de l'examen ${ }^{40}$. Dans les années 1870 , le nombre total de brevets obligatoires décernés aux filles égale celui des brevets décernés aux garçons. À la fin de la période, il devient supérieur. L'augmentation du nombre des brevets de premier ordre semble relever d'une autre logique. En effet, alors que plusieurs de ces brevets sont décernés par les commissions, 
l'inspecteur d'académie indique en 1877, dans son rapport sur la situation de l'enseignement primaire dans le département de la Somme, que seules deux institutrices brevetées sur 109 en sont titulaires ${ }^{41}$. Il y a là, à l'évidence, de la part de certaines candidates, non pas le désir de s'engager dans une carrière primaire, mais la recherche d'un signe de distinction, reconnaissant un niveau d'études que le secondaire ne peut leur offrir ${ }^{42}$.

Les motivations des candidats et candidates n'apparaissent pas dans nos sources, mais l'acharnement avec lequel la plupart tentent d'obtenir l'examen montre combien la pression familiale et sociale peut être forte et les enjeux vitaux. Certains semblent même prêts à de lourds sacrifices pour obtenir ce diplôme. Pour la majorité, la préparation à l'examen exige une présence à l'école au moins jusqu'à 18 ans, âge requis pour pouvoir se présenter devant la commission ${ }^{43}$. Il faut donc rechercher les meilleures stratégies possibles pour une préparation sérieuse et un succès rapide. Comme le montre la scolarité des candidats et candidates, il existe un certain nombre d'établissements primaires et secondaires qui participent activement à cette préparation.

21 La voie royale est connue. Elle existe pour les garçons depuis 1831 avec l'ouverture de l'école normale à $\mathrm{Amiens}^{44}$, mais son accès est limité. L'obligation pour les élèvesmaîtres de se présenter aux épreuves à la fin de leurs années d'études donne rapidement son orientation à l'école normale: la formation s'y confond très rapidement avec la préparation à l'examen pour l'obtention des brevets de capacité. La lecture des programmes d'enseignement confirme cette orientation, parfois dénoncée, de même que les dotations nombreuses en livres pour la bibliothèque et en matériel pédagogique. Les élèves-maîtres sont entraînés pour l'épreuve, et, avant 1850, le passage de la première à la deuxième année s'opère en fonction des espérances de réussite ultérieures à l'examen. Ces conditions expliquent l'excellence des résultats des normaliens. Elles contribuent, plus largement, à l'élaboration de la culture primaire, en étroite relation avec les exigences de l'examen.

Pour les autres candidats et candidates, les voies sont multiples et les parcours parfois longs, difficiles et douloureux, lorsqu'ils se terminent par un échec définitif et l'abandon. Les chances de réussite passent souvent par un séjour de quelques mois dans un pensionnat primaire ${ }^{45}$. Deux exemples illustrent le rôle de ces établissements, le plus souvent privés. Louis Berle, fils d'un ménager domicilié à Morlancourt, élève à l'école communale, subit plusieurs échecs au concours d'entrée à l'école normale et à l'examen pour le brevet aux sessions de 1841 et 1842. Il entre alors comme interne au pensionnat primaire de Monsieur Volland à Amiens et réussit, en août 1843, son entrée à l'école normale. Deux ans plus tard, il obtient le brevet élémentaire. Quelques années plus tard, Pierre Camus, fils d'un cultivateur de Ribemont-sur-Ancre, échoue, lui aussi, aux différentes sessions des années 1847, 1848 et mars 1849. Trois à quatre mois au pensionnat primaire de Monsieur Lencauchez à Corbie lui assurent le succès au brevet élémentaire, en août 1849. Ces deux exemples sont significatifs de la place prise par quelques pensionnats dans la stratégie familiale des candidats au brevet. Certains de ces établissements comme ceux de MM. Volland à Amiens, Lencauchez à Corbie, Nortier à Abbeville, pour les garçons, ou de $\mathrm{M}^{\text {lles }}$ Horville et Leroy à Amiens, pour les filles, proposent à la fois une préparation au concours d'entrée à l'école normale et à l'examen des brevets, et fonctionnent comme de véritables " cours normaux ». Leurs plans d'études sont ambitieux et alignés sur les programmes des épreuves. Ils font 
apparaître l'existence d'une classe accueillant des élèves de 15 à 18 ans, dont les plus âgés exercent parfois des fonctions de sous-maîtres. Ainsi, M. Follet, instituteur libre au Bosquel, autorisé à tenir un pensionnat primaire dans son école depuis 1848 , déclare-til dans un rapport à l'inspecteur départemental en octobre 1856, qu'il a pour l'aider « un jeune homme de 19 ans [...] aspirant au brevet de capacité et un moniteur de 17 ans, également aspirant au brevet $»^{46}$. Dans un autre rapport, daté de novembre 1855, Donzeur, à la tête d'un pensionnat primaire communal à Brutelles, déclare que trois de ses pensionnaires « se destinent à la carrière d'instituteur » et se félicite

que «leur zèle, leur aptitude et leur bonne conduite lui donnent la certitude qu'il formera encore de très bons élèves pour l'école normale $\aleph^{47}$. Même impression dans les pensionnats de filles. $\mathrm{M}^{\text {lle }}$ Horville souligne «le succès qu'ont obtenu plusieurs de ses élèves qui se sont présentées devant les commissions d'examen $»^{48}$. Si les taux de réussite sont plus faibles que ceux des normaliens, 20 à $40 \%$ des inscrits aux sessions (mais beaucoup plus pour les filles), la persévérance les conduit finalement sur la liste des admis.

Ainsi la préparation à l'examen ouvre-t-elle un espace de concurrence entre les établissements primaires. Un véritable marché se constitue, largement dominé par le secteur libre. Le secondaire n'y échappe pas. Les institutions et pensions secondaires, devenues après 1850 les écoles secondaires libres, jouent en effet le même rôle que les pensionnats primaires, même si c'est à un moindre degré. Autorisées à ouvrir une classe primaire, elles y scolarisent parfois des élèves de 15, 16 ans et plus, qu'elles préparent pour l'entrée à l'école normale, et surtout confient les petites classes à des sous-maîtres, souvent anciens normaliens, qui se présentent aux examens.

Les écoles communales retiennent également des élèves âgés, qui se préparent aux épreuves auprès du maître d'école. Ils représentent la moitié des candidats au brevet. Dans ces classes essentiellement rurales, les futurs candidats doivent soulager la tâche des instituteurs et recevoir ainsi une initiation professionnelle par imitation. La formation savante ne dépasse pas, dans ce cas, la simple révision des programmes de la division supérieure. Les manuels d'examen pour les différents brevets, diffusés depuis les années 1830 , leur sont certainement d'une aide très précieuse ${ }^{49}$.

Les cours publics communaux mis en place par la municipalité d'Amiens participent aussi à la préparation du brevet de capacité. En effet, les contenus abordés les rapprochent parfois des exigences de l'examen. Des cours d'arithmétique, de géométrie, de physique-chimie et de dessin linéaire, dispensés le plus souvent par des professeurs du collège royal, puis du lycée, sont ouverts au public scolaire. Les registres d'inscription pour l'année 1852-1853 montrent la présence d'élèves âgés de plus de 10 ans venant de l'école mutuelle d'Amiens, du pensionnat primaire de Volland, mais aussi d'écoles communales ${ }^{50}$. En 1876, des cours municipaux sont spécialement créés pour préparer les jeunes filles en littérature française, histoire et géographie, sciences physiques et naturelles, dessin et anglais.

\section{Le déroulement des examens}

La pratique des examens peut être approchée par la lecture des procès-verbaux rédigés par le secrétaire de la commission et des rapports envoyés aux autorités ${ }^{51}$. Ceux-ci témoignent des exigences des examinateurs, renseignent sur les épreuves et sur les 
performances des candidats. Ils permettent d'identifier les savoirs exigés des futurs maîtres.

La pratique évolue, répondant à la volonté de normalisation et d'uniformisation des autorités. Des prescriptions officielles définissent de plus en plus précisément la nature des épreuves. Si le début des années 1850 constitue un réel tournant de ce point de vue, la période Duruy amorce véritablement des changements profonds quant au caractère même de l'examen. La place prise par de nouvelles disciplines et par les épreuves facultatives destinées à l'obtention d'un brevet complet ou de premier ordre semble fournir la justification à ces évolutions.

\section{Les examens avant 1850}

Le procès-verbal de la session d'août 1843 témoigne du déroulement des examens pendant la période $1833-1850^{52}$. Les travaux de la commission d'instruction primaire convoquée par un arrêté rectoral du 3 juillet 1843 commencent le 21 août 1843 à 7 heures du matin et s'achèvent le 29 à 6 heures du soir. Après avoir examiné les 65 candidats au brevet élémentaire puis les 7 aspirants au brevet supérieur (du 21 au 24 août à midi), le jury se préoccupe des examens des élèves-maitres et du concours d'entrée à l'école normale (du 24 août après-midi au 27 au soir). Les 28 et 29, il organise les épreuves pour les 11 aspirantes institutrices. La session s'achève le 29 août au soir. À l'intérieur de ce calendrier, la commission jouit d'une grande liberté. Le procèsverbal précise que deux heures sont consacrées, au début de la session, de 7 à 9 heures, à une réunion préparatoire pour "arrêter la marche des examens". Il s'agit pour les examinateurs de choisir les sujets, notamment le texte de la dictée, de fixer les critères de correction, d'exclusion et d'admission, le système de notation, de décider ou non la mise en place de sous-commissions, chargées notamment des épreuves orales. Car si les instructions officielles sont précises sur les contenus, malgré l'absence de véritable programme (sauf pour la musique) ${ }^{53}$, elles le sont moins sur la nature des épreuves et le niveau d'exigences souhaité. La commission le regrette parfois, le recteur aussi. Dans une lettre adressée au ministre après la session des trois commissions de l'académie d'Amiens, en 1847, le recteur se plaint des dysfonctionnements et réclame plus d'uniformisation, demandant "d'égaliser autant que possible les conditions de délivrance du brevet de capacité, lequel doit avoir en tous lieux la même signification $»^{54}$.

Quatre épreuves écrites d'admissibilité sont successivement proposées aux candidats et aux candidates : une dictée d'orthographe, une page d'écriture, des questions de calcul et, à partir de $1837^{55}$, une composition de style ${ }^{56}$. La dictée d'orthographe est l'épreuve centrale. De son succès ou de son échec dépend la réussite à l'examen. L'épreuve a lieu immédiatement après l'ouverture de la session et elle est extrêmement sélective : à la session d'août 1843, $40 \%$ des candidats sont éliminés après la correction. Une bonne orthographe est en effet une exigence prioritaire. Dès 1816, les instructions officielles exigent de chaque candidat qu'il écrive "sous la dictée un morceau assez étendu, et renfermant assez de difficultés pour qu'on puisse juger jusqu'à quel point le sujet écrit avec exactitude et correction $»^{57}$. Le texte est proposé par un membre de la commission et semble de sa composition. Avec l'introduction de la rédaction française de deux heures, épreuve également difficile et sélective pour les candidats, et la préférence donnée à un sujet sur table pour le calcul plutôt qu'à une interrogation orale, c'est 
l'écrit qui occupe la première place dans l'examen. Sa maîtrise constitue bien l'une des priorités pour les examinateurs.

Les candidats admis aux épreuves orales se présentent devant des sous-commissions pour être interrogés sur l'instruction morale et religieuse, la grammaire, l'arithmétique, l'histoire, la géographie, les sciences, la musique, les méthodes d'enseignement ${ }^{58}$. À la session d'août 1843 , ce sont 46 candidats qui sont interrogés durant trois jours. Là aussi, le jury se montre sévère : « dix des aspirants au brevet élémentaire sont successivement ajournés par l'extrême faiblesse ou la nullité de leurs réponses aux questions qui leur sont posées ». Mais l'oral sanctionne beaucoup moins que l'écrit.

31 La distinction entre les candidats au brevet élémentaire et au brevet supérieur se fait durant les épreuves. Le premier type de diplôme n'est pas indispensable pour obtenir le second. Les procès-verbaux montrent que les épreuves écrites ne sont pas distinguées, les candidats composent en même temps mais les exigences sont différentes. La dictée et la composition de style sont notées plus sévèrement, des énoncés de problèmes différents sont parfois proposés. Un candidat refusé au brevet supérieur peut se voir attribuer par la commission le brevet élémentaire.

Le procès-verbal montre que les prescriptions ministérielles sont respectées en matière de programme. La maîtrise de la langue française avec des épreuves d'orthographe et de grammaire, la lecture, une bonne pratique des trois grandes formes d'écriture (cursive, ronde et bâtarde), la résolution de problèmes mathématiques simples, montrant la maîtrise des quatre opérations, la connaissance du système légal des poids et mesures, une instruction morale et religieuse solide, quelques notions générales d'histoire et de géographie (à partir de 1833) constituent un ensemble de connaissances de base, le socle exigé de tout futur enseignant du primaire. Il faut compter aussi avec la rédaction d'une composition et le dessin linéaire. À ces savoirs élémentaires, le brevet supérieur, réservé à une minorité de candidats, normaliens pour la plupart, ajoute d'autres disciplines comme les sciences physiques, l'histoire naturelle, la géométrie, la musique, les langues vivantes, l'histoire et la géographie de la France.

\section{Les examens après 1850}

33 À partir des années 1850, les rapports d'examen portent la marque des profonds changements introduits dans la législation. Ceux-ci concernent à la fois le déroulement des épreuves, le fonctionnement des commissions et la nature même du brevet. Le procès-verbal d'août 1851 évoque « les matières relatives à l'enseignement obligatoire " et la possibilité d'être reçu pour " la partie obligatoire ${ }^{59}$. Il cite également un candidat voulant aussi « être interrogé sur toutes les épreuves spécifiées dans la deuxième partie de l'article 23 de la loi du 15 mars 1850 ». Le rapport de juillet 1858 signale des postulants «voulant une mention spéciale sur quelques-unes ou l'ensemble des matières de la deuxième partie $»^{60}$. La loi du 15 mars 1850 a modifié en effet les exigences attendues des futurs maîtres. Son article 23 réorganise les contenus d'enseignement de l'école primaire en distinguant les disciplines obligatoires et les matières facultatives qui peuvent éventuellement s'y ajouter. Les degrés élémentaire et supérieur sont supprimés. Le maintien de deux brevets distincts ne se justifie plus. Un seul subsiste et les instructions officielles le rappellent sans cesse. Ce brevet, dit obligatoire (de second ordre pour les filles) ${ }^{61}$ est allégé de quelques disciplines: 
l'histoire, la géographie, le dessin linéaire et le chant. En revanche, il peut être complété par une mention spéciale, concernant l'interrogation sur une, plusieurs ou la totalité des matières facultatives pouvant être enseignées à l'école ${ }^{62}$, si les candidats en font la demande et s'ils réussissent l'épreuve ${ }^{63}$.

Alors que le brevet obligatoire ne reçoit que peu de modifications significatives au cours des décennies 1850 et 1860 (introduction d'une épreuve d'histoire et géographie par la circulaire du 6 mai 1867), sa partie facultative est l'objet d'une attention ministérielle toute particulière et connaît ainsi une évolution. De simple complément du brevet obtenu après des interrogations orales à l'époque de Fortoul (1852-1856), elle se transforme de fait, après les réformes de Duruy (1863-1869), en un brevet à part entière, dit brevet complet (ou brevet de premier ordre pour les candidates), décerné lorsque sont acquises toutes les matières. Jules Ferry officialise son existence par le décret du 4 janvier 1881, en rétablissant deux brevets de capacité de l'instruction primaire.

Les rapports des sessions d'examen témoignent de ce processus. Celui de juillet 1858 fait référence, pour la première fois à Amiens, à des épreuves écrites en histoire et géographie, en sciences physiques et en histoire naturelle pour les candidats à la partie facultative. L'écrit prend ainsi, comme pour la partie obligatoire, la première place dans l'examen des connaissances en ces matières ${ }^{64}$. La session de mars 1867 marque une nouvelle étape. Y sont évoquées, à propos des aspirants à la partie facultative, des épreuves écrites et orales dans quatre séries. Le rédacteur insiste sur la longueur des compositions et fait explicitement référence à l'article 16 de l'arrêté du 3 juillet 1866, précisant l'ordre et la durée des épreuves ${ }^{65}$. Les rapports qui se succèdent à partir de 1867 montrent que certains candidats s'inscrivent à la deuxième partie du brevet pour des séries différentes lors de sessions successives. L'élévation des exigences se traduit en effet par une complexification de la tâche des examinateurs et une préparation plus soutenue des candidats, qui peuvent postuler plusieurs fois pour enlever, session après session, les quatre séries de la partie facultative. Le brevet se trouve ainsi valorisé, notamment lorsqu'il est complet. On comprend dès lors le rôle qu'il peut jouer comme diplôme sanctionnant et reconnaissant un haut niveau d'études, notamment pour les filles.

D’autres évolutions sont significatives depuis le début des années 1850. Sur le plan institutionnel, les autorités renforcent leur contrôle sur le déroulement et les modalités de l'examen, dans un souci affirmé d'uniformisation, d'efficacité et de justice. L'initiative des examinateurs s'est vue limitée par les décisions de l'arrêté du 15 février 1853 et les instructions du 8 mai 1855. Leur travail s'en trouve modifié. Les sessions ne concernent plus que l'obtention du brevet de capacité. Il n'est plus question des examens des normaliens et du concours d'entrée à l'école normale. Deux moments sont bien identifiés dans la pratique des commissions, celui de l'examen au brevet obligatoire, puis celui des épreuves facultatives pour les candidats en ayant fait la demande au moment de leur inscription. L'examen reste public, mais uniquement pour les garçons. L'ordre des épreuves est fixé réglementairement. Les quatre compositions écrites du brevet obligatoire se succèdent la première journée de la session, en commençant par la dictée. Leur durée (une heure par devoir) et leur notation sont précisées ${ }^{66}$. Les conditions d'ajournement sont explicitées. interrogés, d'abord en lecture, dans un recueil de morceaux choisis (cette lecture est 
accompagnée de questions de compréhension), puis sur le catéchisme et l'histoire sainte; ils doivent ensuite analyser une phrase au tableau noir et répondre à des questions d'arithmétique et sur le système métrique. Le texte du 3 juillet 1866 rappelle que des questions «sur les procédés d'enseignement des diverses matières seront adressées aux candidats". Les aspirants répondent immédiatement aux questions posées, sans temps de préparation. Vingt minutes sont consacrées à chacune des épreuves (arrêté du 3 juillet 1866), soit environ 1 heure 20 par candidat. Les procèsverbaux ne sont pas très précis sur le déroulement de ces interrogations. Celui relatif à la session de mars 1867 indique qu'à partir de « 7 heures du matin la commission interroge successivement, suivant l'ordre de la liste de mérite dressée la veille, et par série de 5, 10 des 17 aspirants, lesquels subissent la totalité des épreuves orales » et qu'à « 5 heures du soir la commission lève la séance et remet au lendemain l'examen des 7 aspirants suivants ».

Ensuite, la commission procède à l'examen des candidats inscrits pour les matières facultatives. Ceux-ci sont nécessairement brevetés, soit depuis la veille, soit lors d'une session précédente. Les épreuves écrites durent une journée. Après correction, les candidats admissibles sont interrogés à l'oral sur les différentes matières demandées. Le même scénario s'applique aux candidates lorsque vient leur tour. On voit que le parcours est devenu particulièrement éprouvant. Rares sont les candidats qui enlèvent, à une même session, les quatre séries proposées, et rares sont les aspirantes qui réussissent l'ensemble des épreuves du brevet de premier ordre.

À partir de 1862, les examinateurs perdent également l'initiative du choix des sujets de composition. En effet, l'arrêté du 27 août confie cette responsabilité au recteur. Il s'agit, là encore, d'éviter de trop grandes disparités entre les commissions en instituant des sujets académiques. L'analyse des contenus des épreuves, à travers la lecture des textes de devoirs proposés, montre un changement évident à partir du ministère Duruy et surtout à partir des années 1870. Cette évolution est sensible dans le choix des textes de dictée $^{67}$, dans les sujets de composition de style et dans la place prise par l'histoire dans les épreuves ${ }^{68}$. Ainsi, à côté de Fénélon et Bossuet, incontournables dans les années 1850-1860, d'autres auteurs apparaissent dans les extraits choisis. Des thèmes nouveaux sont retenus, comme les voyages, la nature et les paysages, et surtout des récits de l'histoire de France $^{69}$. Les sujets de composition française connaissent la même évolution. Les thèmes religieux, autour d'épisodes célèbres de l'Ancien et du Nouveau Testament, cèdent la place aux évocations historiques, notamment à celles de personnages illustres. La comparaison avec les sujets donnés dans d'autres académies en 1879 montre que ces changements ne sont pas spécifiques à l'académie d'Amiens. $75 \%$ des compositions de style invitent les élèves à rédiger sur un sujet portant sur l'histoire de France et $5 \%$ sur l'enseignement de cette discipline à l'école ${ }^{70}$. L'importance de l'histoire grandit, à l'oral des brevets obligatoire et complet (à partir de 1867) et à l'écrit du brevet complet, mais aussi dans les textes de dictée et les compositions françaises proposées aux candidats.

\section{Le brevet de capacité et la culture primaire}

Les examens du brevet ont une autre fonction. Autour des normes d'excellence qu'ils instituent, ils révèlent les performances des candidats et rendent compte du degré d'efficacité des stratégies adoptées. Ils sanctionnent en effet un niveau de 
connaissances acquis pendant une ou plusieurs années de préparation à l'école normale, dans un pensionnat primaire, dans une école communale. Ce bagage constitue la base d'une culture primaire, enrichie pour une minorité accédant au brevet complet.

\section{Les performances des candidats}

Bien que le fonds archivistique de la Somme conserve peu de traces des travaux des candidats, il est possible, à la lecture des rapports et des relevés de notes, de dégager quelques tendances. Comme on l'a vu, l'école normale est la voie royale. Dans les résultats des sessions d'été, les normaliens occupent les toutes premières places. Ils sont particulièrement brillants en mathématiques. Leurs performances en orthographe sont supérieures, en moyenne, à celles des autres candidats. En revanche, en composition de style, ils se heurtent, comme tous les aspirants, à des difficultés. À la session d'août 1864, seuls 3 normaliens sur les 11 reçus ont plus de la moyenne dans cette épreuve, et en août 1866, un seul. Les autres candidats ne déméritent pas. Ils n'hésitent pas à affronter les normaliens à la session de juillet-août, mais sans grand succès. Ils se classent, lorsqu'ils sont admis, derrière les élèves de l'école normale, sauf exception. De ce point de vue, la session de mars leur est plus favorable. Cependant rien ne nous permet d'affirmer que les examinateurs y sont moins exigeants. C'est plutôt leur persévérance qui semble le moteur de leur réussite, puisqu'ils passent plusieurs fois l'examen, alors que les normaliens ne s'y présentent, en règle générale, qu'une seule fois.

À l'évidence, les deux épreuves les plus redoutables restent la dictée d'orthographe et la composition de style. L'une et l'autre demandent beaucoup d'efforts. La rédaction est particulièrement difficile. Les remarques des examinateurs et les notes attribuées montrent une correction sévère. Les garçons réussissent moins bien que les filles. Le rapporteur de la session de mars 1854 signale que les candidats « s'exerceront surtout à la composition écrite que jusque là ils ont beaucoup trop négligée. Ils savent aujourd'hui que cette épreuve est pour eux la plus redoutable, qu'elle est décisive et que cette fois encore elle a été fatale à 16 candidats $»^{71}$. En revanche, lit-on dans le rapport de la session de mars 1852, les filles « ont brillé en instruction religieuse et en composition écrite: plusieurs pages de style sont remarquables par le choix des expressions, l'enchaînement des idées, la délicatesse des sentiments $»^{72}$. Même les normaliens éprouvent des difficultés en dictée et en composition française. Ce sont bien les disciplines d'excellence des examens et celles qui sélectionnent le plus les candidats.

Dans les années 1870 , le discours des examinateurs se fait plus critique à propos de certains sujets proposés, qui font davantage appel à la mémorisation qu'à la réflexion. Ainsi à Amiens en mars 1876, à propos du sujet de style, le secrétaire écrit que la commission souhaite dorénavant « une question de pédagogie ou simplement de bon sens, afin de forcer les candidats à réfléchir et à exprimer ce qui vient d'eux-mêmes, plutôt qu'à rapporter ce qu'ils ont retenu de la lecture ou de l'étude d'un livre d'histoire » et qu' « en histoire et en géographie, comme en instruction religieuse, les réponses sont généralement exactes; mais on voit que la mémoire a pris presque toute la part, sans que la raison y ait été assez mise en jeu ${ }^{73}$. La question des langues vivantes semble également importante aux yeux des examinateurs. De plus en plus de candidats, et surtout de candidates, s'efforcent d'obtenir une mention sur leur brevet 
sanctionnant des compétences en langues, l'anglais et l'allemand principalement, mais aussi, pour quelques postulants, l'italien et l'espagnol ${ }^{74}$. L'épreuve, introduite en 1866 , doit être précisée. La même commission de mars 1866 déclare « qu'il serait nécessaire que la version fût un peu plus accessible qu'elle ne l'est généralement. Souvent empruntée à la poésie, la version renferme des allusions classiques échappant pour la plupart à la connaissance des aspirants; la traduction dès lors en devient très difficile et les contresens abondent: prise dans le domaine des choses ordinaires de la vie, elle présenterait beaucoup plus de chance de succès aux candidats sérieux ». Cet exemple nous semble bien illustrer le processus de construction de savoirs primaires par la pratique de l'examen. Ils doivent se définir d'abord par leur caractère utile et élémentaire. Les examinateurs tentent, là aussi, de réaffirmer la frontière avec la culture classique.

\section{Les composantes de la culture primaire}

La culture religieuse reste fondamentale à l'école primaire. Elle est orale. Si le maître est de moins en moins l'auxiliaire du curé, on attend toujours de lui qu'il soit capable d'enseigner efficacement ce qui demeure tout au long du siècle la première discipline à l'école. La connaissance par les candidats au brevet de l'Ancien et du Nouveau Testament est précise et détaillée ${ }^{75}$. Elle requiert un exercice de mémorisation par un apprentissage sous forme de questions-réponses. La lecture du latin dans un psautier ou un livre d'office est exigée.

Les candidats, futurs maîtres, accèdent également à la maîtrise de la langue française et à une culture littéraire. Mais la frontière avec le secondaire est réaffirmée. Il s'agit plus pour le primaire de privilégier la connaissance de l'orthographe et des principes de base de la grammaire qui l'accompagne, et d'approcher les textes et les auteurs par la lecture d'extraits. La culture classique lui reste étrangère. Le dessin d'ornement et d'imitation, le plain-chant, la musique sont abordés d'un point de vue pratique. Il n'est jamais fait référence à des oeuvres et à des artistes. L'histoire et la géographie sont également, et de plus en plus, constitutives de ce savoir.

Quant à la culture scientifique et technique, elle est essentiellement utilitaire. Il s'agit de pouvoir enseigner aux élèves les règles de calcul indispensables dans la vie quotidienne d'adultes. Dans ce domaine, le brevet complet permet d'atteindre un niveau relativement élevé, comparé à l'enseignement secondaire, avec des connaissances en physique, en chimie, en histoire naturelle, en géométrie, en dessin linéaire, en arpentage et nivellement.

Les stratégies sociales et familiales mises en place témoignent de ce nouveau regard porté sur l'école, de l'adhésion à cette logique d'examen qui ouvre, parce qu'elle repose sur le seul mérite intellectuel, des perspectives nouvelles pour tous. Elles s'inscrivent dans un contexte de bouleversements économiques et sociaux qui affectent aussi les campagnes, et favorisent la mobilité. Comment, en effet, expliquer la persévérance des candidats, sinon par la perspective de cette promotion? Une préparation sérieuse se révèle d'ailleurs généralement payante. Ainsi $30 \%$ des brevetés le deviennent après avoir subi un échec, $20 \%$ réussissent au troisième essai et $10 \%$ obtiennent le brevet à la quatrième tentative. Au-delà, les candidats abandonnent, sauf exception: quelques réussites existent cependant après 7,8 ou 9 tentatives. Le passage par l'école normale assure, en revanche, un succès rapide et brillant. Il permet aussi l'accès à une culture 
primaire supérieure qui ouvre sur d'autres horizons professionnels. En fait l'école normale et le brevet sont indissociables, ils se définissent l'un par rapport à l'autre. Les normaliens se préparent aux épreuves pendant tout leur cursus, le plan d'études qu'ils suivent se définit en référence aux épreuves de l'examen.

La session d'examen prend fin après plusieurs jours de travaux. Les brevetés peuvent postuler pour une école. Il leur reste à faire leurs preuves face aux élèves. Ceux qui ont échoué envisagent, pour la plupart, un nouveau semestre de préparation, peut-être en l'accompagnant d'un changement d'établissement. Les examinateurs, après avoir achevé les formalités administratives, peuvent avoir le sentiment de la mission accomplie. Session après session, ils participent à la formation des nouvelles générations d'instituteurs et d'institutrices.

Il y a bien eu, en effet, une "mutation radicale $»^{76} \mathrm{du}$ personnel de l'enseignement primaire entre le début et la fin du XIX siècle. À l'invention de l'école contemporaine a répondu l'invention de son instituteur. L'étude du corpus archivistique repéré dans le département de la Somme montre que l'institution d'un brevet de capacité de l'instruction primaire, obtenu après un examen obligatoire dont les exigences ont été sans cesse accrues, lance une dynamique et crée un espace dans lequel s'est dessiné progressivement ce nouveau modèle du maître du primaire.

Les instituteurs et institutrices brevetés sont de plus en plus instruits et leur niveau d'instruction de plus en plus homogène. Un véritable corps enseignant primaire, voulu par les autorités, s'est constitué dès avant Jules Ferry. Reconnus pour leurs savoirs et leurs compétences, issus pour leur quasi totalité du monde rural ${ }^{77}$, ils sont devenus des maîtres éclairés, mieux armés pour entreprendre la mission d'instruction et d'éducation du peuple qui leur est confiée. Ce nouveau statut, ils le doivent à la nécessité de se soumettre à un examen de plus en plus exigeant et difficile, pour la réussite duquel ils tentent de trouver les meilleures stratégies. La voie royale du passage par l'école normale n'est pas acquise pour tous, loin de là. Les échecs sont nombreux. Cette dimension sociale, psychologique et affective, nous la mesurons bien dans ces parcours reconstitués de candidats dont les noms reviennent plusieurs fois sur les registres d'inscription à l'examen.

51 L'étude confirme d'autre part l'existence de deux cultures primaires distinctes qui se constituent autour de brevets de capacité aux exigences différentes (élémentaire et supérieur à l'époque Guizot; obligatoire ou simple, comportant ou non une partie facultative, et brevet complet, après 1850) et autour de deux publics. Les normaliens réalisent les meilleures performances et réussissent aux brevets supérieur et complet. La formation disciplinaire qu'ils reçoivent à l'école normale est solide et atteste d'un niveau de connaissances élevé. Mais à partir des années 1860, la concurrence des candidats de certains pensionnats primaires ou certaines écoles élémentaires est réelle. La présence de ces grands élèves auprès de certains maîtres, leurs résultats aux épreuves montrent qu'il existe, à côté de l'école normale, d'autres lieux spécifiques pour préparer l'examen. Dans cette culture primaire, la place de l'écrit et la maîtrise de la langue (rôle de la dictée et de la composition) sont essentielles. La laïcisation des épreuves et l'apparition d'une morale civique et patriotique, dans les années 1860, et surtout à partir de Victor Duruy, annoncent les ruptures des années Ferry.

52 Les commissions d'examen jouent un rôle déterminant dans le processus qui conduit à l'invention de l'instituteur. Les examinateurs constituent un groupe relativement stable, compétent, homogène, instruit et cultivé, mobilisé sur les questions 
d'instruction et d'éducation. Leurs parcours montrent des personnalités locales, toutes amiénoises, de premier plan. Formés dans les humanités et la culture classique, ils ont à l'évidence le souci d'instruire le peuple, mais aussi la volonté de maintenir la frontière entre deux ordres d'enseignement et deux cultures. Le face à face, lors des sessions d'examen, entre les examinateurs et les candidats est aussi le reflet de l'ordre social qui distingue et oppose la bourgeoisie et le peuple. Deux mondes se rencontrent alors: celui des membres des jurys appartenant à la bourgeoisie des talents, à l'élite cultivée et urbaine, à qui on confie la tâche de sélectionner les futurs instituteurs, de définir les contours et les limites du savoir et de la culture primaires, et le monde des candidats et candidates, issus, pour la plupart d'entre eux des milieux populaires de la campagne, des petits métiers ruraux, et aspirant à s'élever aussi bien socialement que culturellement.

\section{NOTES}

1. Les Frères des écoles chrétiennes bénéficient d'un dispositif particulier. L'article 109 du décret précise qu'ils seront brevetés (il s'agit d'un brevet d'institution, sans exigence d'examen), et que les supérieurs " pourront être membres de l'Université ».

2. Toutes les références aux prescriptions officielles proviennent du recueil des textes officiels rassemblés par 0 . Gréard, vice recteur de l'académie de Paris, dans un ouvrage référence en sept tomes: La législation de l'instruction primaire en France depuis 1789 jusqu'à nos jours, recueil des lois, décrets, ordonnances, arrêtés, règlements, décisions, avis, projets de lois, publié, pour la seconde édition entre 1889 et 1902, par la librairie Delalain. Le décret du 17 mars est cité dans le tome 1 , 1789-1833, p. 199.

3. O. Gréard, op. cit., t. 2, 1833-1847, p. 10.

4. Idem, t. 3, p. 609.

5. Instructions du 19 juin 1820 pour les maîtresses de pension et d'études; ordonnance du 22 décembre 1837 pour les surveillantes des salles d'asile; ordonnance du 18 novembre 1845 pour les sous-inspecteurs primaires et les directeurs d'école normale, décret du 29 juillet 1850 pour les inspecteurs primaires d'arrondissement.

6. Voir, sur ce thème, G. Rouet: L'Invention de l'école : l'école primaire sous la Monarchie de Juillet, Nancy, Presses universitaires de Nancy, 1993, et F. Jacquet-Francillon: Instituteurs avant la République, Presses universitaires du Septentrion, 1999.

7. La première session d'examen de la période Guizot a lieu le 28 août 1833 à Amiens, devant une commission provisoire. Elle est ouverte aux seuls normaliens. La nomination officielle de la première commission d'instruction primaire a lieu en février 1834. Les années 1833, 1834 et 1835, voient la convocation d'une troisième session en automne. Elle disparaît ensuite.

8. Amiens est chef-lieu académique de mai 1808 à septembre 1848, puis de mars 1850 à juin 1854 . Entre septembre 1848 et mars 1850, et après juin 1854, l'académie d'Amiens est supprimée et le département de la Somme est rattaché à l'académie de Douai.

9. Pour les candidates, depuis mars 1837.

10. Archives départementales de la Somme (AD Somme), $2 \mathrm{~T} 23$.

11. O. Gréard, op. cit., t. 1, p. 271. 
12. Dans la Somme, la commission a été installée par un arrêté préfectoral du 29 août 1819 . Voir AD Somme, $4 \mathrm{~K}$ 4. L'article 4 de l'arrêté prévoit que « les postulantes devront se présenter devant le jury d'examen institué à Amiens pour le département de la Somme » et l'article 5 arrête sa composition : 1 membre du Conseil général, 1 membre du tribunal civil d'Amiens, 1 inspecteur de l'académie, 1 ecclésiastique, 1 conseiller de préfecture.

13. L'enseignement primaire féminin passe sous le contrôle du ministère de l'Instruction publique avec l'ordonnance du 21 avril 1828 (article 21). Jusque cette date, il dépend du ministère de l'Intérieur.

14. O. Gréard, op. cit., t. 2.

15. Idem, p. 10.

16. Circulaire du 13 août 1836. Le recteur de l'académie d'Amiens fait le choix de commissions départementales uniques dans son ressort. La plupart des académies font de même.

17. Le règlement du 19 juillet 1833 prévoit la nomination d'une commission de 7 membres dont 3 devront être pris parmi les membres de l'Instruction publique. Le recteur (ou l'inspecteur d'académie) préside la commission. Le proviseur ou le censeur et un professeur du collège royal, le principal du collège communal, un régent en font partie. La loi du 15 mars 1850 maintient les 7 membres et impose la présence d'un inspecteur d'arrondissement pour l'instruction primaire, d'un ministre du culte professé par le candidat et de deux membres de l'enseignement public ou libre (art. 46).

18. À partir de 1864, bien que la présence de l'inspecteur d'académie soit toujours attestée, un autre examinateur préside.

19. L'ordonnance du 26 février 1835 institue un inspecteur spécial de l'instruction primaire par département. Le règlement du 27 février 1835 prévoit que l'inspecteur primaire est membre de droit des commissions et qu'il y exerce les fonctions de secrétaire.

20. L'ordonnance du 13 novembre 1837 crée les sous-inspecteurs primaires ( 1 ou 2 par département). Ils ne font pas nécessairement partie des commissions (décision du 31 août 1838) mais peuvent y être adjoints sur proposition du recteur. C'est le cas à Amiens.

21. La Commission des lettres est instituée à Amiens le 18 janvier 1816. Elle est chargée jusqu'en 1848 de l'examen des candidats au baccalauréat pour l'académie.

22. AD Somme, $2 \mathrm{~T} 2$, registre du personnel de l'académie d'Amiens, 1827-1855; 60T396189, registre du personnel, 1865-1869; 2T411 à 2T416, Commission des lettres.

23. Circulaire du 6 août 1833. Les commissions sont, en effet, chargées d'examiner tous les normaliens qui doivent, en fin d'études, obtenir le brevet de capacité, mais aussi, de 1833 à 1850 $\mathrm{du}$ concours d'entrée à l'école normale et des examens de passage de $1^{\mathrm{re}}$ et $2^{\mathrm{e}}$ année.

24. Celle-ci est créée à Amiens en mai 1817 pour favoriser le développement de l'enseignement mutuel.

25. Les commissions nommées à Laon et à Beauvais par le ministre en 1840 et 1846 ont la même composition qu'à Amiens. On y trouve, à peu près, un tiers de membres du collège et $30 \%$ appartenant à la magistrature, à la médecine ou aux grandes administrations techniques.

26. Commissions nommées en 1837, 1840, 1843 et 1849, et repérées dans le Bulletin universitaire, d'octobre 1837 à septembre 1839 pour la $2^{\mathrm{e}}$ commission triennale, de mars 1840 à novembre 1841 pour la $3^{\mathrm{e}}$, de janvier à mars 1843 , pour la $4^{\mathrm{e}}$, de janvier à avril 1846 pour la $5^{\mathrm{e}}$ et de janvier à août 1849 pour les dernières. Soit au total, 297 commissions. Le secondaire représente $30 \%$ des examinateurs. Les personnalités extérieures à l'instruction publique et aux écoles professionnelles rassemblent $33 \%$ des effectifs.

27. Voir, sur ce thème, P. Rosanvallon : Le moment Guizot, Paris, Gallimard, 1985.

28. La loi du 15 mars 1850 institue le brevet de capacité de l'enseignement secondaire, indispensable à toute personne non titulaire du baccalauréat et voulant ouvrir un établissement secondaire libre. L'examen se déroule devant un jury composé de 7 membres nommés par le 
ministre. À Amiens, le ministre nomme une commission académique le 27 septembre $1850 ; 4$ membres désignés, Hardouin, Rigollot, Pollet et Spéry, appartenaient jusqu'alors aux commissions primaires.

29. La loi du 15 mars 1850 supprime les sous-inspecteurs primaires; ils sont remplacés par un inspecteur primaire dans chaque arrondissement.

30. La congrégation religieuse de la Sainte Famille est installée à Amiens depuis 1817. Elle joue le rôle d'école normale pour les filles, puisqu'elle forme des maîtresses pour les écoles rurales.

31. AD Somme, 2T146. Rapport au recteur sur les travaux de la commission pour les examens des candidates, session d'août 1848 (9 septembre 1848).

32. AD Somme, $2 \mathrm{~T} 144$. Dans le compte rendu de la session de mars 1839 au ministre, le recteur de l'académie d'Amiens déclare, le 22 juin 1839: "La pénurie d'institutrices dans laquelle se trouvent les trois départements du ressort académique faisait un devoir aux commissions d'être plus indulgentes à l'égard des aspirantes. C'est ainsi qu'il faut s'expliquer le nombre relativement plus grand des aspirantes admises et non par le plus haut degré d'instruction de celles-ci ».

33. Voir l'étude de R. Lemoine : La loi Guizot, son application dans le département de la Somme, 1933.

34. D'après l'enquête de 1833. Les relevés effectués aux Archives nationales (F17*151) montrent que sur 985 instituteurs repérés, 959 sont brevetés.

35. AD Somme, 60T3729. En 1850, 90 \% des instituteurs sont communaux et $98,6 \%$ sont laïcs (959 instituteurs recensés). Les institutrices, au nombre de 262, sont congréganistes à $60 \%$, et communales à $61 \%$.

36. AD Somme, 60T3458. Le nombre d'écoles dirigées par un instituteur a diminué. Il est de 919 (98\% sont laïcs et publics). Ils sont maintenant secondés par 202 adjoints ( $80 \%$ de laïcs et de publics). L'ensemble de ce personnel est breveté (sauf 41 congréganistes adjoints).

37. ADS, 60T3458. Les 469 écoles de filles sont aux deux tiers congréganistes. Mais près de $75 \%$ des adjointes sont religieuses et non brevetées.

38. Le terme de brevet complet est utilisé lorsque le candidat, reçu au brevet simple, a satisfait à l'ensemble des matières ou des séries proposées dans la partie facultative. Pour les filles, il s'agit alors du brevet de premier ordre. Le brevet de second ordre correspond au brevet obligatoire puis simple.

39. Victor Duruy est ministre de l'Instruction publique de 1863 à 1869 .

40. AD Somme, 60T3518. En 1870, le département de la Somme compte 409 écoles de filles, dont 289 , soit $71 \%$, congréganistes.

41. Bulletin départemental de la Somme, $\mathrm{n}^{\circ}$ 234-235-236, 1878, Bibliothèque nationale (8LC10 417 (7).

42. F. Mayeur a bien souligné cette réalité : « l'engouement pour les brevets est l'une des réalités du Second Empire. L'habitude de couronner les études des jeunes filles par un brevet primaire a commencé sous la Monarchie de Juillet » (L'éducation des filles au XIXe siècle, Hachette, 1979).

43. L'ordonnance du 12 mars 1831 fixe à 18 ans accomplis l'âge minimum des candidats. Celle du 23 juin 1836 fixe à 20 ans celui des filles. Le décret du 20 juillet 1850 ramène à 18 ans l'âge minimum des candidates, celui du 2 mai 1870 à 16 ans.

44. L'école normale des filles ouvre ses portes en 1876.

45. La législation, depuis 1812 , précise qu'une école primaire peut s'adjoindre un pensionnat après autorisation et sous certaines conditions. Leur nombre est variable dans le département de la Somme, tout au long du xixe siècle. On en compte de 50 à 80 en moyenne, d'inégale importance.

46. AD Somme, 99T150.

47. AD Somme, 99T150.

48. AD Somme, $2 \mathrm{~T} 379$.

49. Lamotte, Meissas, Michelot: Manuel des aspirants au brevet de capacité de l'enseignement primaire élémentaire et supérieur, Hachette, 1836 (nombreuses rééditions) ; Jullien, Meissas, Sainte-Preuve : Manuel d'examen pour le brevet de capacité à l'usage des aspirants et des aspirantes, Hachette, 1854 ; 
Lenient: Guide des aspirants et aspirantes aux divers brevets de capacité pour l'enseignement primaire, librairie P. Dupont, $1879,5 \mathrm{e}$ édition.

50. Dans le journal local L'Étoile de la Somme, du 7 octobre 1855, une annonce précise que «les chefs d'institution qui désireront faire suivre les cours communaux par leurs élèves sont invités à les faire inscrire à la mairie, où des cartes d'admission leur seront délivrées. Les élèves devront avoir au moins 10 ans pour être admis aux cours d'arithmétique, de géométrie, de mécanique et de dessin linéaire, 13 ans et demi pour suivre les cours de physique et 15 ans pour suivre celui de chimie » (AD Somme 733 PER 1).

51. Les Archives départementales de la Somme possèdent une collection à peu près complète de rapports sur les sessions depuis mars 1836. Il manque les comptes rendus des sessions d'août 1836, des années 1841 et 1842, de mars 1846, mars 1849 et les années 1855 à 1857 et 1860 à 1863.

52. AD Somme, $2 \mathrm{~T} 145$.

53. Arrêté du 29 mars 1836.

54. AD Somme, 2T146, lettre du recteur de l'académie d'Amiens au ministre, du 18 mars 1847.

55. Règlements du 28 juin 1836 pour les filles, du 11 octobre 1836 pour les garçons, et du 1 er octobre 1840.

56. Les commissions décident parfois que la seule dictée jouera le rôle d'épreuve d'admissibilité. Le calcul est parfois renvoyé à l'oral.

57. Instructions du 14 juin 1816, dans O. Gréard, op. cit, tome 1. À Amiens, la commission réunie pour la session d'août 1838 décide que tout candidat qui aura fait plus de trois fautes, celles de ponctuations comprises, ne pourra plus concourir pour le brevet du degré supérieur et que, pour être admis à continuer les épreuves pour le brevet élémentaire, un candidat ne devra point avoir fait plus de huit fautes, celles de ponctuations comprises (AD Somme, 2T144).

58. Le règlement du 28 juin 1836 impose aux candidates, dans son article 3, « une leçon orale d'une demi- heure ». L'arrêté du 11 octobre 1836 étend cette mesure aux candidats. Dès la session d'août 1837, la commission de la Somme constate la difficulté de la mise en place de cette épreuve et « décide que la note de la leçon orale sera donnée d'après l'ensemble de l'examen ».

59. AD Somme, 60T3273 : procès-verbal de la session d'août 1851.

60. AD Somme, 99T147.

61. Arrêté du 31 décembre 1853.

62. Il s'agit de la $2^{\mathrm{e}}$ partie de l'article 23 de la loi du 15 mars 1850 : «ces matières facultatives sont: l'arithmétique appliquée aux opérations pratiques, les éléments de l'histoire et de la géographie, des notions de sciences physiques et de l'histoire naturelle, applicables aux usages de la vie, des instructions élémentaires sur l'agriculture, l'industrie et l'hygiène, l'arpentage, le nivellement et le dessin linéaire, le chant, la gymnastique. Pour les filles s'ajoutent les travaux à l'aiguille ".

63. Le candidat qui s'inscrit pour la partie obligatoire peut demander, lors de la même session, à être interrogé sur une matière facultative ou plusieurs. Une mention en sera portée sur le brevet. En revanche, un candidat déjà breveté lors d'une précédente session ne peut postuler que pour l'ensemble des matières de la $2^{\mathrm{e}}$ partie de l'article 23 de la loi du 15 mars 1850 (arrêté du 15 février 1853). La circulaire ministérielle du 11 août 1858, qui revient sur cette question, déclare « qu'un instituteur ne saurait, en effet, être admis, de session en session, devant une commission d'examen, à seule fin de faire constater chaque fois, jusqu'à épuisement du programme facultatif, sa capacité sur l'une des matières qui y sont comprises. Il a donc été décidé que les candidats déjà brevetés ne pourraient être interrogés que sur l'ensemble de ces matières ». À partir de 1862, le brevet facultatif, lorsque sont acquises toutes les disciplines concernées, est appelé brevet complet.

64. L'arrêté du 27 août 1862 officialise ces compositions écrites pour les aspirants au brevet complet. Il exige un récit emprunté à l'histoire nationale, une question de géographie, deux 
questions sur les notions de sciences physiques et de l'histoire naturelle, trois questions sur l'arpentage, le nivellement et le dessin linéaire, trois questions sur l'agriculture, l'industrie et l'hygiène. Pour les aspirantes, le brevet de premier ordre comprend les mêmes épreuves écrites sauf les questions sur l'arpentage, le nivellement, l'agriculture, l'industrie et l'hygiène, mais plus la musique.

65. L'arrêté du 3 juillet 1866 rassemble les épreuves écrites de la partie facultative en quatre séries : $1^{\text {re }}$ : l'arithmétique et la géométrie, appliquées aux opérations pratiques; le dessin

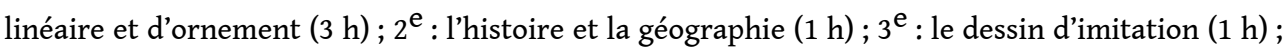
$4^{\mathrm{e}}$ : langues vivantes (thème et version : $1 \mathrm{~h}$ ).Les épreuves orales, pour chacune des séries, comprennent: $1^{\text {re }}$ : arithmétique; tenue des livres; éléments de géométrie; arpentage, nivellement, dessin linéaire et d'ornement; chant $(1 \mathrm{~h}) ; 2^{\mathrm{e}}$ : éléments d'histoire et de géographie; notions de sciences physiques et d'histoire naturelle applicables aux usages de la vie ; instructions élémentaires sur l'agriculture, l'industrie, l'hygiène ; la gymnastique $(1 \mathrm{~h}) ; 3^{\mathrm{e}}$ : dessin d'imitation (une demie heure) ; $4^{\mathrm{e}}$ : langues vivantes (une demi heure).

66. Fortoul précise dans les instructions du 8 mai 1855 le système de notation : « les commissions adopteront désormais un système de signes exprimant la valeur intrinsèque de chacune des épreuves. Ces signes... seront chiffrés de 0 à 10. Tout candidat qui n'aura pas obtenu, pour les quatre épreuves écrites, une moyenne de 20 points ne sera pas admis aux épreuves orales. La nullité d'une épreuve sera un cas absolu d'exclusion. Des points seront également donnés pour les épreuves orales, et le brevet ne pourra être accordé qu'à ceux des candidats qui, pour l'ensemble des épreuves, auront obtenu un minimum de 40 points pour les aspirants et (en raison des travaux d'aiguille) de 45 pour les aspirantes ».

67. L'arrêté du 15 février 1853 précise que le texte de la dictée « sera pris par le président dans un livre classique ".

68. L'arrêté du 15 février 1853 indique que la composition de style sera "un récit emprunté à l'histoire sainte ou une lettre relative à la tenue d'école » (0. Gréard, t. 3, p. 516). Celui du 3 juillet 1866 évoque, pour l'exercice de style, une composition d'histoire d'une heure (0. Gréard, t. 4, p. 107).

69. Les textes des dictées retrouvés dans les archives départementales ou dans les bulletins de l'instruction primaire témoignent de cette ouverture à de nouveaux auteurs: Perrault, Marmontel, Molière, Buffon, Marivaux, Racine, La Bruyère, La Rochefoucault et même, Balzac, Jules Simon, Augustin Thierry.

70. Le sujet de composition de style donné en août 1854 à Amiens propose aux candidats de réfléchir au sujet suivant: "Vous raconterez comment les israélites, sous la conduite de Josué, prirent possession de la terre promise ». Ces thèmes de l'Ancien Testament reviennent fréquemment. Ainsi, en août 1859, les garçons sont invités à composer autour de l'histoire de David et Salomon, et les filles sur « la démarche de Moïse auprès du pharaon pour l'engager à permettre aux enfants d'Israël de sortir du pays». À partir de 1869, ce sont les grands personnages de l'histoire de France, comme Clovis, Charlemagne, Saint Louis, Louis XIV, Bonaparte, mais aussi des événements comme les Croisades, qui sont sollicités. Les sujets de l'année 1879, pour l'ensemble de la France, sont répertoriés dans le Recueil des sujets de composition donnés aux examens du brevet de capacité pendant les années 1879-1880, de E. D’Ollendon, Paris, 1881.

71. AD Somme, 60T3636.

72. AD Somme, 60T3273.

73. AD Somme 60T3264.

74. AD Somme, 60T3514, 99T149, 60T3499, 99T403051, 60T3272, 60T3262. Sur 102 candidats et candidates recensés, $60 \%$ s'inscrivent en anglais (53\% pour les garçons et $72 \%$ pour les filles), 
$30 \%$ en allemand ( $32 \%$ pour les candidats et $28 \%$ pour les candidates), $10 \%$ en italien et espagnol (15\% des garçons).

75. Les procès verbaux des sessions indiquent parfois les notes obtenues par les candidats aux épreuves orales depuis 1858. Les moyennes obtenues sont relativement élevées. Ainsi, en mars 1876 , les candidats obtiennent 7,10 pour l'instruction religieuse (6,19 pour la lecture, 6,70 pour l'analyse grammaticale, 6,74 pour le calcul et 6,60 en histoire-géographie). Le classement est le même pour les candidates (AD Somme, KZ1856, 6OT403501). À partir de 1864, dans notre corpus, les questions données à l'oral sont reportées. Elles montrent la nécessité pour les candidats de connaître précisément les principaux événements de l'histoire sainte et le catéchisme.

76. Selon l'expression de A. Prost: Histoire de l'enseignement en France, 1800-1867, Paris, A. Colin, 1968, p. 136.

77. Les candidates au brevet sont, durant toute la période étudiée, davantage d'origine urbaine que les garçons.

\section{RÉSUMÉS}

Depuis 1816, une obligation nouvelle s'impose à tout futur maître du primaire, celle de posséder un brevet de capacité obtenu après le succès à un examen. Cette obligation est étendue aux institutrices laïques en 1819 et aux membres des congrégations enseignantes masculines en 1831. Mais la loi du 28 juin 1833 marque une étape essentielle dans l'histoire de ces examens. En plaçant les commissions d'instruction primaire au cœur du dispositif, en rendant les examens publics, en exigeant des connaissances solides, Guizot, puis ses successeurs, font de l'examen une institution reconnue. Les candidats et candidates sont de plus en plus nombreux à s'y préparer, à l'école normale ou dans d'autres établissements ; ils ambitionnent de conquérir le seul diplôme qui ouvre aux carrières de l'enseignement primaire et certifie un ensemble de savoirs constitutifs d'une culture primaire. Sa possession, obtenue après la réussite à des épreuves de plus en plus difficiles, participe bien, entre Guizot et Ferry, à la construction du nouveau corps des instituteurs primaires.

From 1816 onwards, any would-be primary school teacher had to submit to a new obligation : that of holding a diploma after sitting an examination. This obligation applied to secular female teachers in 1819 and also to members of the male teaching congregations in 1831. However, the law of June 28, 1833 became a milestone in the history of those examinations. Guizot, and his successors later on transformed this examination into a recognized practice by giving a central place to primary education commissions, by making the examinations public and by requiring sound knowledge. There was a growing number of male and female candidates preparing for the entrance exam to teacher training schools or other establishments. It was their ambition to obtain the only diploma that offered a career in primary education and that guaranteed all the requisites for primary school culture and knowledge. Obtaining the diploma after passing increasingly difficult examinations contributed to the setting up of the new body of primary school teachers.

Wer vorhatte, als Elementarschullehrer sein Brot zu verdienen, musste seit 1816 eine wichtige Voraussetzung erfüllen: er musste einen Befähigungsnachweis erwerben und sich dafür einer Prüfung unterziehen. Seit 1819 galt diese Vorschrift auch für zukünftige Volksschullehrerinnen 
im Laienstand, seit 1831 für männliche Mitglieder der in der Laienbildung tätigen Ordensgemeinschaften. Das Gesetz vom 18 Juni 1833 stellt einen wichtigen Schritt in der Geschichte dieser Prüfungen dar. Guizot setzte eine spezielle Prüfungskommission ein, ließ die Öffentlichkeit zu den Prüfungen zu und verlangte den Kandidaten solide Kenntnisse ab, wodurch der Befähigungsnachweis zu einer allgemein anerkannten Einrichtung wurde. Immer mehr männliche und weibliche Prüflinge unterzogen sich ihr in Normalschulen oder vergleichbaren Einrichtungen; immer ging es darum, das einzige Diplom zu erwerben, das eine Laufbahn als Elementarschullehrer möglich machte und die Beherrschung eines soliden Grundwissens attestierte. In der Zeit zwischen Guizot und Ferry trug dieses Zertifikat, das durch immer schwierigere Prüfungen erworben werden musste, entscheidend zur Ausbildung des neuen Berufsstandes des Volksschullehrers bei.

INDEX

Index géographique : France, Somme (département)

Index chronologique : XIXe siècle

Mots-clés : brevet de capacité, corps enseignant, enseignement primaire, examen, instituteur

\section{AUTEUR}

\section{DANIEL TOUSSAINT}

Doctorant

IUFM de l'académie d'Amiens 